\title{
Binding of Artificial Object to Vorticella for a Microsystem Powered by a Microorganism*
}

\author{
Moeto Nagai ${ }^{\dagger}$ and Momoko Kumemura \\ Institute of Industrial Science, The University of Tokyo, \\ 4-6-1 Komaba, Meguro-ku, Tokyo 153-8505, Japan \\ Hiroshi Asai \\ Waseda University, 3-4-1 Okubo, Shinjuku-ku, Tokyo 169-8555, Japan \\ Hiroyuki Fujita \\ Institute of Industrial Science, The University of Tokyo, \\ 4-6-1 Komaba, Meguro-ku, Tokyo 153-8505, Japan. \\ (Received 21 October 2008; Accepted 4 April 2009; Published 6 June 2009)
}

\begin{abstract}
Miniaturization, integration, and functionality of microelectromechanical systems are limited in water. One solution to this problem is to apply the micrometer-scale motors from biological systems. A suitable biological motor is a linear motor found in microorganisms such as Vorticella. We developed a method to attach artificial material onto Vorticellas through streptavidin biotin binding. We biotinylated Vorticellas and coupled them to streptavidin coated particles of $1,2,6$ and $8 \mu \mathrm{m}$ diameter. The binding force of streptavidin and biotin was strong enough to endure a high speed contraction of a Vorticella (several $\mathrm{mm} / \mathrm{s}$ ). This attachment process of artificial material is a crucial step to fabricate a microsystem powered by filaments of Vorticellas. [DOI: 10.1380/ejssnt.2009.673]
\end{abstract}

Keywords: Biological compounds; Biological molecules - proteins; Adhesion; Surface chemical reaction

\section{INTRODUCTION}

Microelectromechanical systems (MEMS) can sense, control, and activate mechanical processes on the micro scale and mainly work in vacuum or air. However, miniaturization, integration, and functionality of MEMS are limited in water. There are two reasons: (1) high voltages to drive MEMS require good insulation between electrodes and, (2) electronic hardware to control MEMS actuators can be easily damaged by water and must be protected; this results in the large size of the total system. These limits the sizes and functionalities of MEMS. One solution to this problem is to apply the micrometerscale motors from biological systems. A microorganism functions effectively underwater, has an actuator and a control system in its cell body, and does not require outer system for control.

A suitable powerful biological motor is a linear motor found in microorganisms such as Vorticella. Vorticella has a filament called as stalk, which contracts and elongates in response to the concentration of calcium ion (Fig. 1). A stalk tethers to a substrate and Vorticella uses a stalk and finishes contraction within $9 \mathrm{~ms}$. Moriyama et al. estimated the maximum contracting force is greater than 55.8 $\mathrm{nN}$ [1]. After cell membrane of a Vorticella is excluded, outer solution reaches inside the sheath of a stalk, and both contraction and elongation of the Vorticella become controllable by changing the concentration of calcium ion in solution [2]. Therefore Vorticella can be used as a linear actuator equipped with a sensor for calcium ion. In order to obtain an effective work, it is indispensable to attach an artificial microobject onto its surface and as-

\footnotetext{
* This paper was presented at International Symposium on Surface Science and Nanotechnology (ISSS-5), Waseda University, Japan, 9-13 November, 2008.

†Corresponding author: moeto@iis.u-tokyo.ac.jp
}
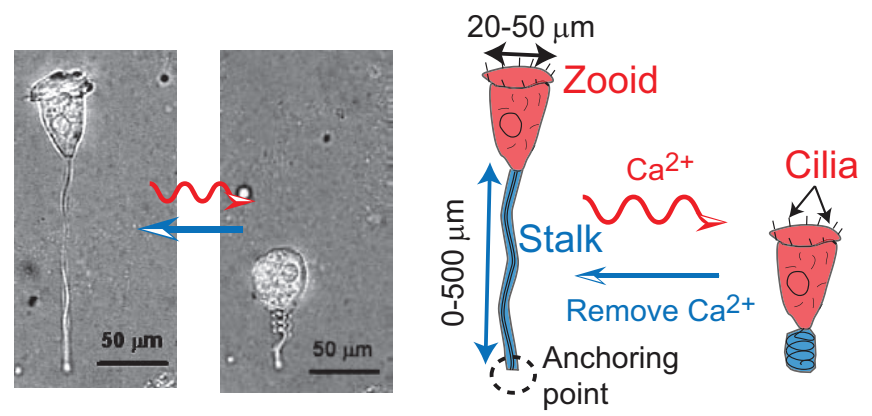

FIG. 1: A vorticella contracts and elongates in response to concentration of calcium ion.

semble a mechanical system. However, the method to bind artificial material to Vorticella has not established yet.

Some methods to bind cells and artificial materials have been reported [3-6]. Since the surface conditions of cells are diverse, attaching methods differs from one case to another. Weibel et al. synthesized peptides with a high affinity for Chlamydomonas reinhardtii and attached the particles onto the cell body [3]. Darnton et al. and Steager et al. coupled microsize objects to Serratia marcescens through affinities of PDMS (Polydimethylsiloxane) [4] and SU-8 [5], respectively. Hiratsuka et al. anchored microobjects to Mycoplasma mobile [6]. They used the property that streptavidin has a strong affinity for a vitamin, biotin. They attached biotin to amino groups on the surface of the cells (this attachment process is called biotinylation) and microobjects were coated with streptavidin. They mixed the objects and the cells, and coupled the microobjects to the cells. As described before, in order to bind artificial material to a cell, it is essential to understand the surface condition of a cell and use a material with an affinity for the cells surface.

We developed a process to attach a microobject onto a Vorticella through an adhesive property that streptavidin 

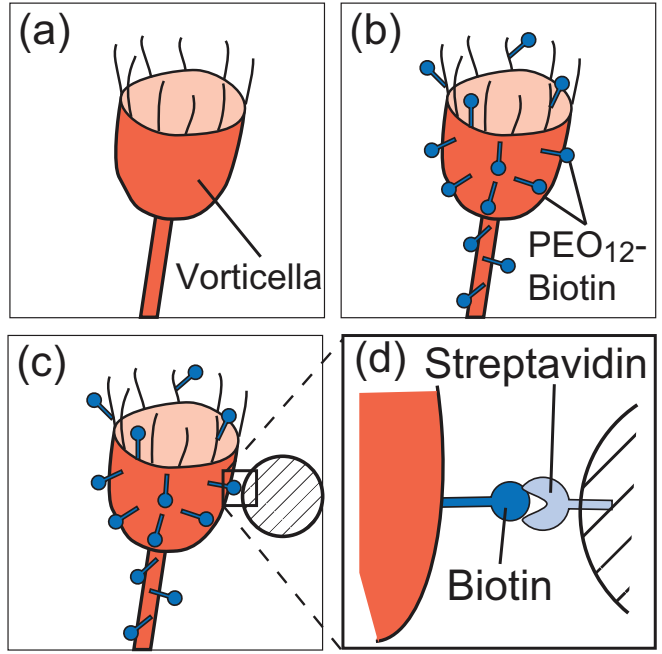

FIG. 2: A schematic diagram to bind artificial material onto a Vorticella. (a) A Vorticella has amino groups on its surface. (b) Biotinylation of Vorticella. Biotin- $\mathrm{PEO}_{12}$-NHS which has amino-reactive group is chemically linked to the Vorticella. (c) Then streptavidin coated particle attaches the Vorticella through streptavidin-biotin binding. (d) The magnified view of the region enclosed by the box in (c).

binds biotin as a first step to realize microsystems driven by harnessed microorganisms. Amino groups existing on the surface of a Vorticella were modified and coated with biotin. Then we introduced streptavidin-coated particles near the Vorticella and attached the particles. We optimized the composition of a buffer solution for Vorticellas to keep them alive and active over two hours.

\section{EXPERIMENTAL}

Vorticella Picta was collected from lake Hattyou in Yoshimi, Saitama. We isolated Vorticella Picta and cultivated them in a culture medium containing bacteria at room temperature, around $25^{\circ} \mathrm{C}$. We kept $18 \times 18 \mathrm{~mm}$ coverslips floating (Matsunami Glass Ind., Ltd., thickness: $0.12-0.17 \mathrm{~mm}$ ) on the culture medium for a few days until a suitable number of Vorticellas attached onto the coverslips. We made a filtered infusion of dry yeast, EBIOS (Asahi Food \& Healthcare Ltd., Tokyo, Japan) as shown below and used it for the culture medium. The medium was infused by autoclaving $2.5 \mathrm{~g} / 1$ of EBIOS in Milli-Q water at $120^{\circ} \mathrm{C}$ for 20 minutes. Then the infused liquid was filtered through a $0.2-\mu \mathrm{m}$ filter unit (NALGENER) Disposable Filter Unit, 566-0020; Capacity: $500 \mathrm{~mL}$ ) to remove solid materials. Prior to use, we diluted this filtered liquid tenfold with Milli-Q water and used as the culture medium.

We assembled a flow chamber and conducted all steps of chemical reaction of Vorticellas and attachment of particle in the chamber. The flow chamber was composed of three parts. (1) Vorticella-attached coverslip, as described above. This part was used as an upper side of the flow chamber. (2) A coverslip of larger size than the upper side $(24 \times 36 \mathrm{~mm}$, Matsunami Glass Ind., Ltd., thickness $0.12-0.17 \mathrm{~mm})$. This part was used for the bottom side of the flow chamber. (3) A spacer, which is sand-


FIG. 3: Optical micrographs of Vorticellas which particles attached through streptavidin-biotin binding. The diameters of each particles are (a) $1 \mu \mathrm{m}$, (b) $2 \mu \mathrm{m}$, (c) $6 \mu \mathrm{m}$, respectively. Red arrows indicate attached particles. Scale bars: $50 \mu \mathrm{m}$.

wiched between two coverslips not to crush Vorticellas. We coated a paper with silicone grease (DOW CORNING TORAY HIGH VACUUM GREASE, Dow Corning Toray Co., Ltd.) to make it adhesive. We pipetted different types of solutions from one side of the fabricated flow chamber and removed it from the other side by capillary action using Whatman \#2 filter paper.

We injected Dryl's solution $\left(2 \mathrm{mM} \mathrm{Na} \mathrm{Citrate}_{2} \cdot 2 \mathrm{H}_{2} \mathrm{O}\right.$, $0.6 \mathrm{mM} \quad \mathrm{NaH}_{2} \mathrm{PO}_{4}, \quad 1.4 \mathrm{mM} \quad \mathrm{Na}_{2} \mathrm{HPO}_{4}, \quad 1.5 \mathrm{mM}$ $\left.\mathrm{CaCl}_{2} \cdot 2 \mathrm{H}_{2} \mathrm{O}\right)$ into the chamber and washed Vorticellas. Then we introduced $0.4 \mathrm{mM}$ NHS-PEO ${ }_{12}$-Biotin (Pierce, 

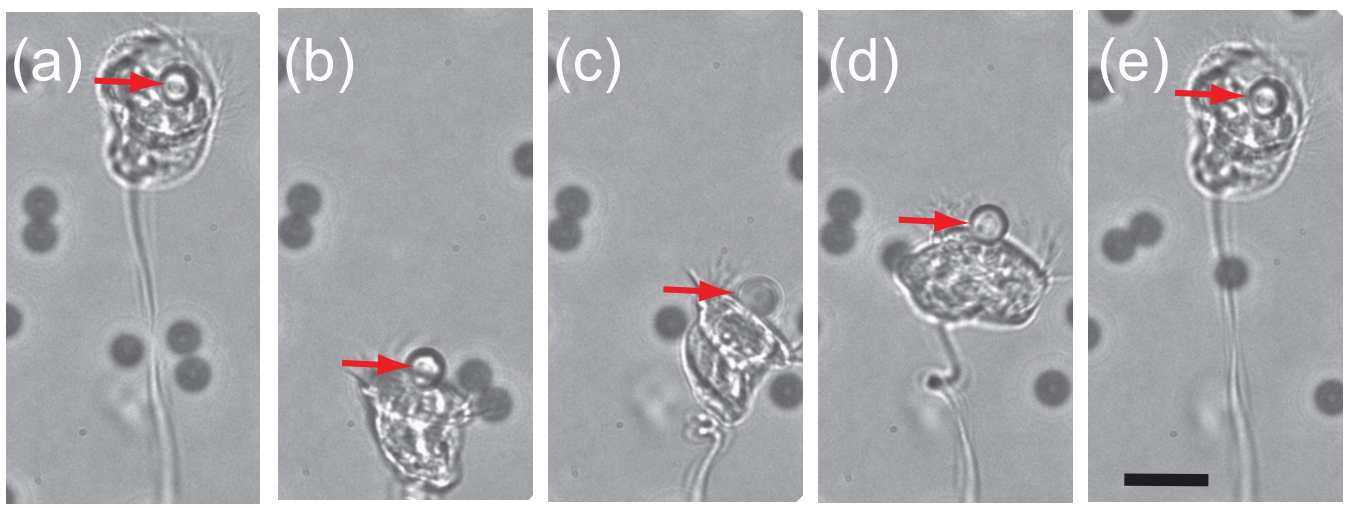

FIG. 4: Time-series optical micrographs of $8 \mu \mathrm{m}$ particle in diameter, which attached to a Vorticella during a contraction and elongation. The location of the particle is almost the same (a) before contraction and (e) after elongation. Scale bar: $20 \mu \mathrm{m}$.

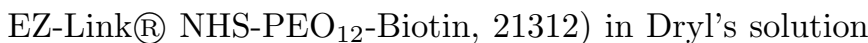
to the chamber and coated biotin tags with Vorticellas from the amino groups on the surfaces of Vorticellas as shown in Fig. 2. We kept Vorticellas in the solution for over $30 \mathrm{~min}$. Here, we prepared two types of samples, biotinylated and non-biotinylated samples in order to examine the effect of biotinylation. After the biotinylation, we injected Coupling Buffer (0.1-0.2\% Bovine Serum Albumin [Sigma], $\mathrm{KCl} 50 \mathrm{mM}, \mathrm{MgCl}_{2} 2 \mathrm{mM}, \mathrm{MOPS}-\mathrm{KOH}$ $10 \mathrm{mM}$ ) to remove the unreacted solution.

We used streptavidin coated particles of four different sizes from two suppliers. Particles of 1,2 and $6 \mu \mathrm{m}$ in diameter were purchased from Polysciences, Inc. (streptavidin conjugated microspheres, 24162, 24160 and 24158 respectively). Particles of $8 \mu \mathrm{m}$ diameter were obtained from micromod Partikeltechnologie $\mathrm{GmbH}$ (micromer $\AA$ ) 01-19-803). Prior to use, we washed the particles with the coupling buffer by centrifuging the particles and discarding the supernatant several times. We adjusted the final concentration of the particles to $0.137,0.063,0.069$, $0.241 \%(\mathrm{v} / \mathrm{v})$ for the particles of $1,2,6$ and $8 \mu \mathrm{m}$ in diameter, respectively.

After we injected the particles into the chamber and mixed them with Vorticellas, we put the flow chamber on an inverted microscope (IX71, Olympus) equipped with an objective lens (Magnification $60 \times$, N.A. $=0.7$, Olympus), and a CCD camera (Resolution $512 \times 512$ pixel, 16 bit, Cascade II 512, Photometrics). We evaluated attachment of the particles on the movements of the particles. When particles follow the motion of Vorticellas, we regard these particles are attached.

\section{RESULTS AND DISCUSSIONS}

We describe a comparison between biotinylated sample and non-biotinylated one in order to measure the effect of biotinylation. In case of biotinylated sample, the particles followed movement of cilia or stalks of Vorticellas. Meanwhile, in case of non-biotinylated sample, we did not observe the particles follow the motions of Vorticellas. The particles moved randomly in the solution. The particles did not attach onto the surfaces of Vorticellas. This comparison between two samples reveals that the biotinylation helps to attach streptavidin particles.

The particles of 1, 2, 6 and $8 \mu \mathrm{m}$ diameter attached Vorticellas and followed their motion (Figs. 3 and 4). Number of attached particles depended on the size of the particle. As the size of the particles decreased, the number of attached particle increased. Vorticellas were alive, and contracted and elongated over two hours in our optimized buffer solution.

Particles of $1 \mu \mathrm{m}$ diameter attached Vorticellas more than ones of other diameters. The particles attached to (1) cilia, (2) cell body and (3) stalk in decreasing order. Most of the Vorticellas had over 20 particles attached. Figure 3(a) shows many particles attach to cilia of a Vorticella and the cilia are tied up. At most 6 particles attached to a single cell body and 3 particles were coupled to a single stalk. Numbers of attached particles depended on the lengths of stalks. There was a tendency that the longer stalk had more attached particles. Since many particles attached the glass surface near the anchoring points, it was hard to observe anchoring points.

Figure 3(b) shows the particles of $2 \mu \mathrm{m}$ diameter coupled to cilia. The particles in the figure followed the motion of the cilia. Number of particles attached to cilia is fewer than half the number of the samples of $1 \mu \mathrm{m}$ in diameter. Some particles attached to cell bodies. Almost all particles were floating during observation.

A particle of $6 \mu \mathrm{m}$ diameter attached to cilia as shown in Fig. 3(c). The particle moved according to the cilia motion. Number of particles was less than the cases of particles of 1 and $2 \mu \mathrm{m}$ in diameter. Cilia had two particles at most. We did not observe particles which attached stalk. Almost all particles sank down to the bottom and number of the attached particles was less than the number of particles of 1 and $2 \mu \mathrm{m}$ in diameter.

A particle of $8 \mu \mathrm{m}$ in diameter attached onto a Vorticella (Fig. 4). The particle (indicated by red arrows) followed the motion of the Vorticella while it contracted and elongated. In the figure, all particles except the attached one were not floating and stayed on the bottom side of the coverslip. The binding force of streptavidin and biotin was strong enough to endure the high speed contraction of a Vorticella (several $\mathrm{mm} / \mathrm{s}$ ). Trace of the attached particle during the elongation is depicted in Fig. 5. Number of particles was less than other particles. We did not ob- 


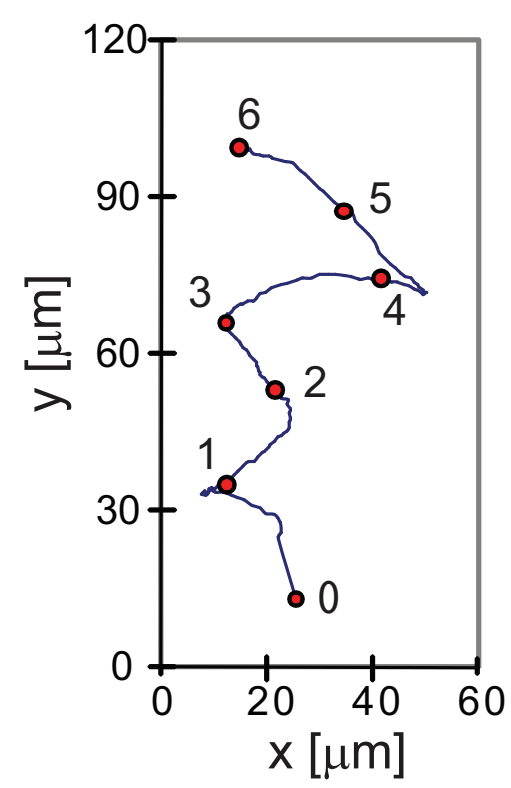

FIG. 5: Time-series trace of the particle which attached to the Vorticella in figure 4 during elongation. Numbers near red points in the view area represent time, $\mathrm{t}[\mathrm{s}]$, from starting point of elongation. At $\mathrm{t}=0 \mathrm{~s}$, the Vorticella fully contracted. From $\mathrm{t}=0 \mathrm{~s}$ to $\mathrm{t}=6 \mathrm{~s}$, the Vorticella gradually elongated rotating the cell body. When $\mathrm{t}=6 \mathrm{~s}$, the Vorticella finished its elongation.

serve particles which attached stalk. Almost all particles were deposited on the coverslip.

The distribution of the particles is explained by contact probability between particle and each region of a Vorticella. Cilia move constantly in the solution generating the flow at the speed of above $2500 \mu \mathrm{m} / \mathrm{s}$ [7] and the contact probability is the greatest. On the other hands, cell body and stalk move less than the cilia and the contact probability is smaller than one of the cilia.

There are two reasons that larger particle is more difficult to attach than small one: (1) Small diameter particles have higher surface area to volume ratio and are easier to attach to Vorticellas than larger particles. (2) The larger particles are more easily precipitated than the smaller particles. When a particle is large, there will be less floating particles. Presuming that we use the same density of par- ticles and we increase the size of particles, sedimentation velocity, which is proportional to the square of diameter increases as shown in Eq. (1). Therefore the larger particles are more easily precipitated than the smaller particles.

$$
v=\frac{d^{2}\left(\rho-\rho_{0}\right) g}{18 \eta}
$$

where $d$ : diameter of particle, $\rho$ : density of particle, $\rho_{0}$ :density of solution, $g$ : gravity acceleration, $\eta$ : viscosity of solution.

It is preferable that artificial material attach to the cytoskeletal structure rather than to fluid molecules inside a cell membrane. This is because the cytoskeletal structure can transmit the contracting and elongating force of Vorticella to attached material more easily than the fluid-like cytoplasm. Therefore, material attached to the cytoskeletal structure can follow the motion of the Vorticella more easily. It is presumed that larger particles need stronger binding forces to stay attached because of the greater viscous force associated with larger particles.

\section{CONCLUSIONS}

We developed a method to attach an artificial material to microorganisms through streptavidin-biotin binding. We biotinylated Vorticellas and coupled streptavidin coated particles of $1,2,6,8 \mu \mathrm{m}$ diameter to them. This method enables us to assemble a device powered by Vorticella. This attachment process of artificial material is a crucial step to fabricate a microsystem powered by the stalks of Vorticellas.

\section{Acknowledgments}

We acknowledge Dr. Isamu Morishita for the identification of Vorticella species. We appreciate Dr. Douglas Weibel at University of Wisconsin-Madison and Dr. Yuichi Hiratsuka at Japan Advanced Institute of Science and Technology for helpful discussion of attachment of artificial material.
[1] Y. Moriyama, S. Hiyama, and H. Asai, Biophys. J. 74, 487 (1998).

[2] W. B. Amos, Nature 229, 127 (1971).

[3] D. B. Weibel, P. Garstecki, D. Ryan, W. R. Diluzio, M. Mayer, J. E. Seto, and G. M. Whitesides, Proc. Natl. Acad. Sci. U.S.A. 102, 11963 (2005).

[4] N. Darnton, L. Turner, K. Breuer, and H. C. Berg, Biophys. J. 86, 1863 (2004).
[5] E. Steager, C.-B. Kim, J. Patel, S. Bith, C. Naik, L. Reber, and M. J. Kim, Appl. Phys. Lett. 90, 263901 (2007).

[6] Y. Hiratsuka, M. Miyata, T. Tada, and T. Q. Uyeda, Proc. Natl. Acad. Sci. U.S.A. 103, 13618 (2006).

[7] M. A. Sleigh and D. Barlow, Trans. Am. Microscopical Soc. 95, 482 (1976). 